| Original | Article |

\title{
Local flap for reconstruction of nasal defect following excision of basal cell carcinoma of nose
}

\author{
Hasib Rahman, Syed Farhan Ali Razib, Abul Kalam Azad, M. A. Mannan, Md. Ashik Anwar Bahar \\ and Md. Mosleh Uddin
}

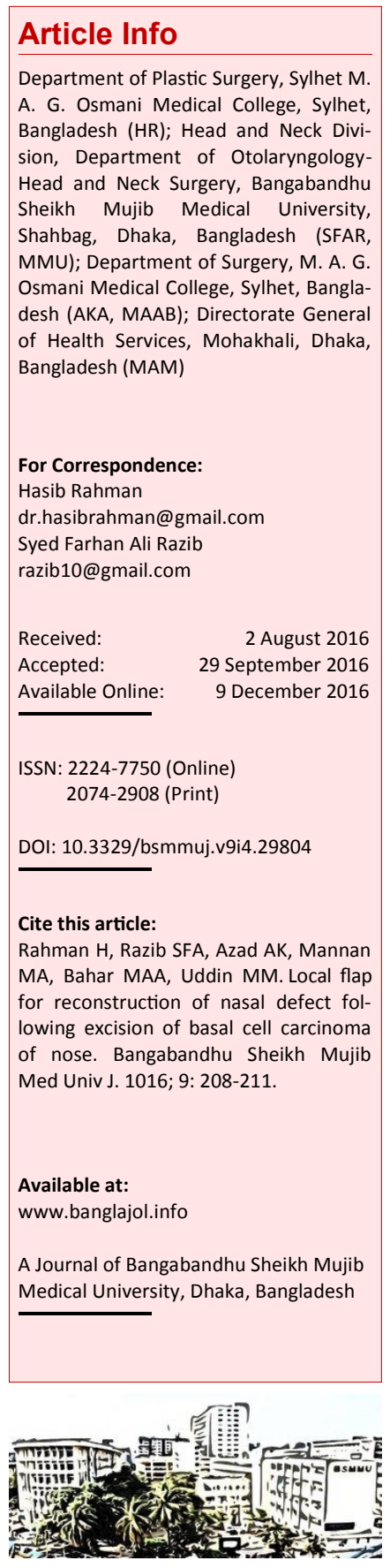

\begin{abstract}
Reconstruction of soft tissue at the nose following excision of basal cell carcinoma is always challenging, because of both functional and aesthetic importance of nose. The local flap is always preferable to skin graft as this produces "like with like" replacement, pliable cover and vascularized tissue over the skeletal framework. In this paper, we discussed six cases of nasal reconstruction with bilobed flap, forehead flap, and nasolabial flap. All flaps survived and the patients had satisfactory outcome.
\end{abstract}

\section{Introduction}

Basal cell carcinoma is the most common nonmelanoma skin cancer.1 About $80 \%$ of all basal cell carcinoma occur on the face, among which $25 \%$ involves the nose due to the cumulative effect of the sunlight.2,3

Surgery is the main mode of treatment in these patients with a goal to achieve a tumor-free margin. Several studies advocated excising the lesion with $0.5 \mathrm{~cm}$ margin to achieve $95 \%$ cure rate. 4 But this creates an aesthetic challenge for the plastic surgeon as nose possesses significant aesthetic and functional importance.

A plastic surgeon should carefully consider a number of characteristics unique to the nose, including the inherent structural complexity of the nose, with convex and concave surfaces in close proximity, the symmetry of the nose, the limited laxity of the nasal skin, and the sebaceous composition of distal nasal skin. Missing parts should be replaced with tissues considering the quality and quantity.

For this, the flap is always preferable than skin grafts as it produces a superior match in color and texture. It has the additional advantage of producing a vascularized soft tissue cover for nasal skeleton and resistant to contractures. $\underline{5}$ The characteristics of skin covering the nose are not homogenous. The skin covering the bony parts is highly movable, while the skin over cartilage parts is thicker, tighter and bound to the cartilage. So, reconstructive options will vary with size, location, skin laxity, age and tissue availability. No single flap can be considered universal for the nasal defect. The common local flaps for nasal reconstruction are the bilobed flap, nasolabial flap, forehead flap and its variations. 6 However, the decision of flap selection is individualized based on the above mentioned factors.

\section{Materials and Methods}

Six patients admitted at the Department of Plastic Surgery, Sylhet M. A. G. Osmani Medical College and Hospital suffering from basal cell carcinoma of the nose from January 2015 to January 2016 were treated with wide excision and reconstruction with local flaps. Four different types of local flaps were used for reconstruction. Age, sex, site and size of the lesion were recorded. A viable flap on discharge was considered as a successful outcome.

\section{Results}

Among the six cases (male 4, female 2), the youngest case was 8 years old and the eldest was 62 years. Classical median forehead flap was done in three cases. Island median forehead flap $(n=1)$, nasolabial flap $(n=1)$ and bilobed flap $(n=1)$ were done. All flaps survived with the satisfactory aesthetic outcome.

Case 1: A 61 year old non-diabetic, nonhypertensive male patient presented with an ulcer with rolled edge, about $1.2 \mathrm{~cm}$ in diameter, over the tip of the nose (Figure 1). 


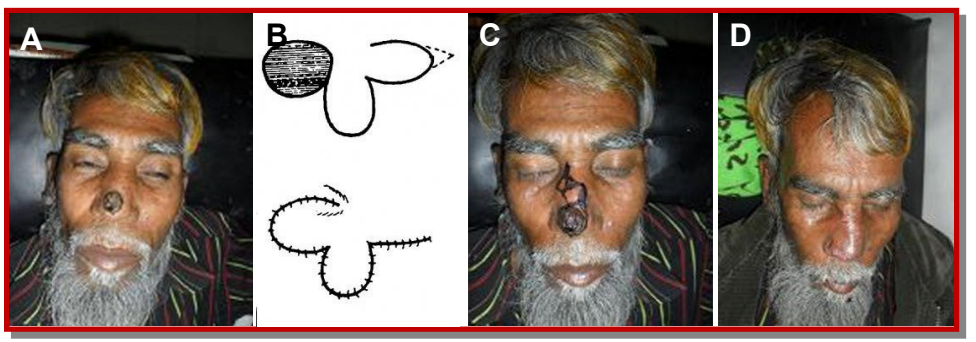

Figure 1: (A) Basal cell carcinoma over tip of nose; (B) Principle of bilobed flap; (C) Bilobed flap designed; (D) Post-operative result

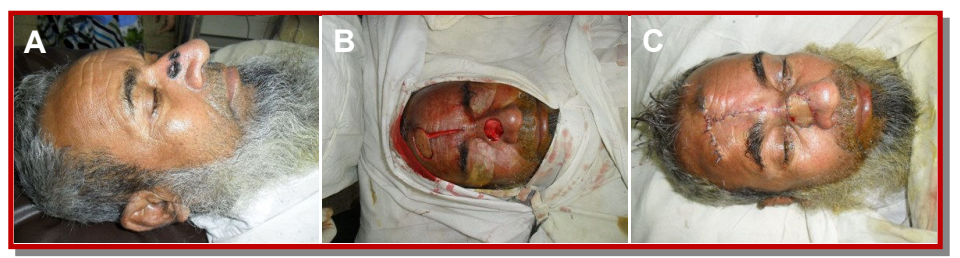

Figure 2: (A) Basal cell carcinoma at the dorsum of the nose; (B) Flap design of the cutaneous island; (C) flap inset with primary closure of the donor site

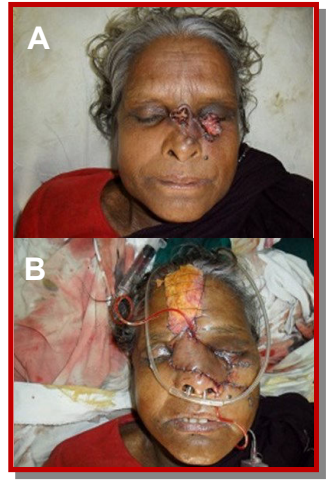

Figure 3: (A) Basal cell carcinoma root of nose and eyelid; (B) flap cover of defect with skin grafted donor site

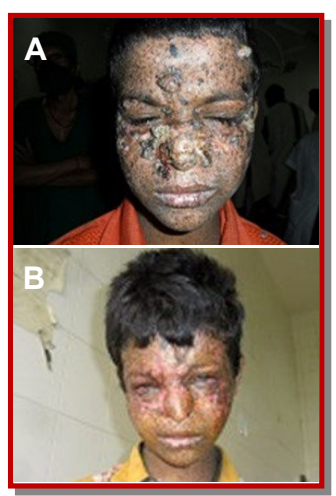
Figure 4: (A) Multiple pearly edge and a necrotic ulcerated center. The basal cell carcinoma floor of the ulcer was covered with slough. It was involving nose and face; confined to the skin and freely movable over the (B) Post-operative ap- deep structures. The cervical lymph node was not pearance

The floor of the ulcer was covered with slough, base indurated but not fixed with underlying lower lateral cartilages. Cervical lymph nodes were not palpable. Incision biopsy confirmed it as basal cell carcinoma.

Surgical procedure: Surgery was performed under general anesthesis and the lesion was excised with $0.5 \mathrm{~cm}$ margin. After excision, lower lateral cartilages were exposed but perichondium was intact. The final defect was about $1.7 \mathrm{~cm}$ in diameter. Bilobed flap was planned to cover the defect. A transposition flap with two lobes with a common semicircular base was designed. Proximal lobe was designed tangentially to the defect over the dorsum of the nose and the distal lobe at $90^{\circ}$ to the initial defect in the upper lateral wall of the nose. The length of the proximal lobe was same as the defect but the width was about $20 \%$ less. The distal lobe was narrower than the proximal, triangular in shape permitting primary closure. Diluted epinephrine (1:100000) was infiltrated and both flaps were raised over nasal framework in submuscular plane. The proximal flap was transposed to cover the primary defect with excision of a dog ear. Distal flap used to cover the defect created by transposition of the proximal lobe with primary closure of distal lobe defect as the skin was loose over lateral wall of the nose. Skin closure was done in two layers.

Case 2: A 56 year old male patient presented with a pigmented lesion occupying dorsum and the right lateral wall of the nose (Figure 2). The lesion was 2.5 $x 1.5 \mathrm{~cm}$ in its maximum diameter, with a rolled, enlarged. Incision biopsy revealed it as basal cell carcinoma.

Surgical procedure: Surgery was performed under local anesthesia (2\% lidocaine with adrenaline in 1:100000 dilutions). The lesion was excised with 0.5 $\mathrm{cm}$ margin. After excision, the upper lateral cartilage of the nose was exposed. An island forehead flap was planned for reconstruction of the nose. A template of the lesion was made and was used to mark the cutaneous island of the median forehead flap on the ipsilateral side of the forehead. A vertical incision was made up to the medial edge of the right eyebrow to expose the flap pedicle. Frontalis and corrugator muscles were included over proximal two third of the flap base. The flap was transposed through a subcutaneous tunnel to cover the defect.

Case 3: A 57 year old female presented with an ulcerated lesion with rolled edge with intervening scar involving root of the nose, upper part of dorsum and left lateral wall of the nose and medial two-third of lower eyelid (Figure 3). Incision biopsy was performed for confirmation of the basal cell carcinoma.

Surgical procedure: The lesion was excised with 0.5 $\mathrm{cm}$ margin. The final defect was $7 \times 4 \mathrm{~cm}$ in its maximum diameter. A median forehead flap based on supratrochlear and supraorbital artery was designed and raised in conventional technique. The flap was transposed to cover the defect. Donor site was skin grafted.

Case 4: A 10 year old boy of xerodermapigmentosum presented with multiple basal cell carcinoma of face and nose (Figure 4). Basal cell carcinoma of the nose, cheek, temporal region were excised. Removal of basal cell carcinoma of the nose resulted in total soft tissue loss over the nose. A median forehead flap with gull wing design was harvested and rotated to cover the defect. Donor site over forehead and rest of the wounds were skin grafted.

Case 5: This patient, 8 year old girl was the younger sister of the case 4 . She had her basal cell carcinoma removed from the nose and lower eyelid (Figure 5). Reconstructive procedure was same as case 4 .

Case 6: A 62 year old male patient presented with a small $0.5 \mathrm{~cm}$ ulcer occupying right ala (Figure 6). Lesion was excised with $0.5 \mathrm{~cm}$ margin and alar skin was replaced with a inferiorly based nasolabial flap. Donor site was closed primarily.

\section{Discussion}

Nose is the commonest site of basal cell carcinoma.2 Excision with $0.5 \mathrm{~cm}$ margin creates a significant defect considering the inadequate pliable tissue preventing primary wound closure in most of the cases. Although skin graft can be done to cover the defects, it produces contour deformity and color 


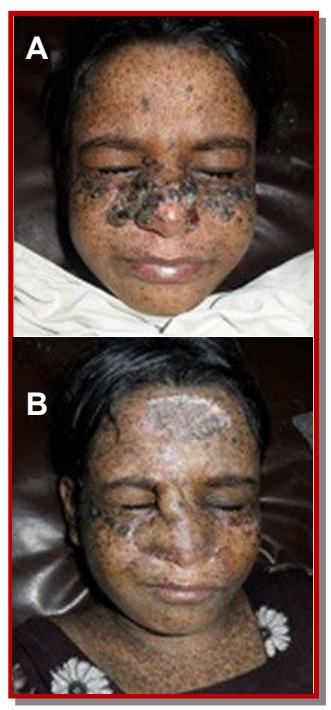

Figure 5: (A) Basal cell carcinoma of the nose, lower eyelid and cheek; (B) Post-operative appearance

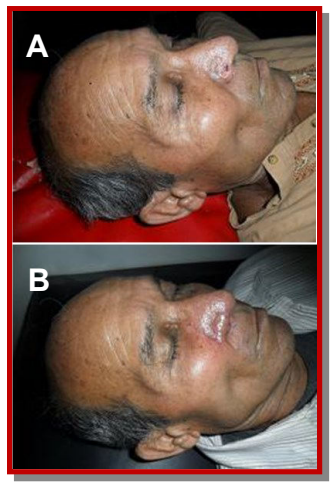

Figure 6: (A) Basal cell carcinoma of the ala of the nose; (B) Nasolabial flap cover of the ala mismatch. Local flaps have the advantages of replacing like with like with superior aesthetic and functional outcome. Most common local flaps for nasal reconstruction are the bilobed flap, forehead flap with its variations and nasolabial flap. But the final choice of flap selection is individualized based on site, size, age, skin laxity and the need of the patient.

Bilobed flap was first described by Esser in 1918, who used it to cover nasal tip. 7 Zitelli modified the design by reducing the rotation angle and it became the workhorse flap for 1-1.5 cm defect of distal and lateral part of the nose, particularly for nasal tip.., 9

Bilobed flap replaces the missing skin in distal part of the nose with similar skin from the proximal area of nose. It is excellent choice to reconstruct the distal part of the nose but it should be designed as well as appropriately sized to prevent secondary motion at the recipient site. The size of the primary and secondary lobe should be equal to minimize distortion and to avoid post-operative alar elevation the donor site of the secondary lobe should be placed perpendicular to alar margin. After considering all the factors the incision should be placed up to the perichondrium as well as periostium before being raised. $\underline{8}$

Bilobed flap is easy to execute, single staged and has good cosmetic results. But it will not be possible to cover the nasal wound more than $1.5 \mathrm{~cm}$ in diameter so larger defects required more complex reconstruction.

Forehead flaps are the mainstay for reconstruction of large cutaneous nose defects. It is a type of fasciocutaneous flap based on supratrochlear (dominant pedicle) and supraorbital (minor pedicle) vessels. Kazanjian advocated primary closure of the forehead donor site. $\underline{10}$ Millard applied a characteristic gull wing design with lateral extension to reconstruct the alar margin in 1960s and 1970s and to get greater flap length he extended the pedicle incision below the brow. $\underline{\mathbf{1 1}, 12}$ Burget and Menick emphasized for aggressive thinning the skin paddle and they also advised narrowing the pedicle base for easier rotation and length and to modify defects to follow aesthetic subunit of the nose. $\underline{\mathbf{1 3 , 1 4}}$

Traditionally the median forehead flap requires two stages for the division of the bridging segment. But it can be transferred as a single stage procedure by deepithelializing the skin of the pedicle and placed it under the glabellar skin to fill the nasal defect which converts the median forehead flap into an island flap. A wide subcutaneous tunnel is advisable to avoid the compromization of vascular pedicle (case 2).

While reconstructing the nasal defect an ipsilateral flap has the advantage to cover the distal border for its effective greater flap length but there may be chance of pedicle base compromization due to its greater arc of rotation. However, a contralateral flap may not reach to the distal end but it has less rotation at the pedicle base. To keep the supratrochlear artery in between the orbicularis oculi and corrugator muscle dissection should be continued under the corrugator muscle.

Forehead flap may be contraindicated because its mobilization sometimes unsuccessful if deep, horizontal scars placed across the base of the forehead. If scars are small and superficial then it is accepted but if extend to galea then it will produce significant barrier to the blood supply through the pedicle.

One of the advantage of nasolabial flap is that can be used to reconstruct the lateral alar defect as it is a 1-step transposition/advancement flap $\underline{15}$ and its donor site is skillfully hidden in the melolabial crease and it should be undermined properly to prevent anatomic distortion. But blunted alar groove and development of trapdoor deformity are some disadvantages of this flap as well.

Excess tissue near the melolabial fold matches with the color and texture of the nose and its underlying fat has a strong tendency to contract. As the melolabial fold has enough skin which can be used to resurface the ala and its nature of contractility is useful to maintain the round expected bulging of the normal ala $\underline{16}$ and natural expression lines of the face may hide the donor site scar.

If the surgical defect is located on the lateral ala or in the area of the alar groove then the nasolabial flap is selected by some authors where incision line of the flap is made directly into the melolabial fold. To make the flap elongated at its donor site on the medial cheek, it should be shortened before transpose (illustrating pivotal restraint). There may be risk of developing unsightly tissue protuberances in the postoperative period due to dog ears in the area of the melolabial fold and immediately superior to the surgical defect over the nose which should be removed generously. $\underline{15,16}$

Nasolabial flap is not suitable to reconstruct tissue defect greater than $3 \mathrm{~cm}$ in diameter in which distant tissues like forehead flap can be a good choice for reconstruction.

\section{Conclusion}

We used bilobed flap for a small defect in the nasal tip, nasolabial flap for alar defects and forehead flap for larger defects needing cover for more than twothird of the nose. As face is very vascular, these flaps in skilled hands are reliable and useful to have better aesthetic and functional result. All our flaps survived and patients had satisfactory outcome. 


\section{References}

1. Netscher DT, Spira M. Basal cell carcinoma: An overview of tumor biology and treatment. Plast Reconst Surg. 2004; 113: 70-94.

2. Ge NN, McGuire JF, Dyson S, Chark D. Nonmelanoma skin cancer of the head and neck II: Surgical treatment and reconstruction. Am J Otol. 2009; 30: 181-92.

3. Boyd AS, Shy Yr, King LE. Basal cell carcinoma in young women: An evaluation of the association of tanning bed use and smoking. J Am Acad Dermatol. 2002; 46: 706-09.

4. Wolf DJ, Zitelli JA. Surgical margins for basal cell carcinoma. Arch Dermatol. 1987; 123: 340-44.

5. Burget GC. Aesthetic restoration of the nose. Clin Plast Surg. 1985; 12: 463-80.

6. Zitelli JA, Fazio MJ. Reconstruction of the nose with local flaps. J Dermatol Surg Oncol. 1991; 17: 18489.

7. Zimany A. The bilobed flap. Plast Reconstr Surg. 1953; 11: 424-34.

8. Cook JL. Reconstructive utility of the bilobed flap: Lessons from flap successes and failures. Dermatol
Surg. 2005; 31: 1024-33.

9. Cook JL. A review of the bilobed flap's design with particular emphasis on the minimization of alar displacement. Dermatol Surg. 2000; 26: 354-62.

10. Kazanjian VH. The repair of nasal defects with a median forehead flap: Primary closure of forehead wound. Surg Gynecol Obstet. 1946; 83: 37.

11. Millard DR Jr. Reconstructive rhinoplasty for the lower half of a nose. Plast Reconstr Surg. 1974; 53 : 133-39.

12. Millard DR Jr. Reconstructive rhinoplasty for the lower two-thirds of the nose. Plast Reconstr Surg. 1976; 57: 722-28.

13. Millard DR Jr. Reconstructive rhinoplasty for the lower two-thirds of the nose. Plast Reconstr Surg. 1977; 59: 876-88.

14. Burget GC, Menick FJ. Nasal reconstruction: Seeking a fourth dimension. Plast Reconstr Surg. 1986; 78: 145-57.

15. Zitelli JA. The nasolabial flap as a single-stage procedure. Arch Dermatol. 1990; 126: 1445-48.

16. Rieger RA. A local flap for repair of nasal tip. Plast Reconstr Surg. 1967; 40: 147-49. 